\title{
Verunsicherung bei Impfungen vermeiden
}

- Nach einer Pressemeldung der KV Rheinland-Pfalz gibt es dort, wie bereits im vergangenen Jahr, für die Impfsaison 2010/2011 keine einheitliche Regelung für die saisonale Influenzaimpfung. So lehnt die dortige AOK in diesem Jahr nicht nur die Kostenübernahme für die saisonale Influenzaimpfung außerhalb der Schutzimpfungsrichtlinien $a b$, sondern verhindert auch noch, dass Krankenkassen, die die Impfung für ihre Versicherten bezahlen möchten, den Impfstoff als Sprechstundenbedarf (SSB) beziehen können. Vertragsärzte in Rheinland-Pfalz müssen deshalb für die Impfungen innerhalb und außerhalb der Richtlinien unterschiedliche Abrechnungsnummern einsetzen und die Impfseren über verschiedene Kanäle beziehen.

\section{MMW-Kommentar}

Hintergrund der Blockade der AOK sind einerseits die üblichen Sparbemühungen, die prospektive Maßnahmen zur Kostenreduktion - wie dies bei Impfungen eindeutig der Fall ist - aus haushalterischen Gründen ignorieren. Hinzu kommt aber ein weiterer Faktor: die Jahr für Jahr bundesweit sehr hohen Verluste beim Impfstoffbezug. In Hessen z.B. werden jährlich für etwa 9 Millionen Euro Impfstoffdosen über den Sprechstundenbedarf bezogen, aber nicht verimpft. Solche Verluste sollen dort künftig durch ein verbessertes Impfmanagement verhindert werden. Die Ärztinnen und Ärzte in Hessen sind seit dem 1.7.2010 gehalten, nur so viel Impfstoff aus Apotheken oder von sonstigen Lieferanten zu ordern, wie sie voraussichtlich auch tatsächlich verbrauchen können. Dies könnte z.B. durch das Einrichten von Impfsprechstunden und/oder den Bezug des Impfstoffes über spezialisierte Versandapotheken erreicht werden. Der Modellversuch in Hessen, der mit allen Kassen vertraglich vereinbart werden konnte, sieht für den besonderen Aufwand, der mit einem solchen Management verbunden ist, ein zusätzliches Honorar vor.

\section{GKV-Finanzen im 1. Halbjahr 2010 immer noch im Plus}

- Während die Krankenkassen im ersten Halbjahr 2009 noch einen Überschuss von 1,2 Mrd. Euro verbucht hatten, betrug der Überschuss im ersten Halbjahr 2010 immerhin noch rd. 112 Mio. Euro. Für das Gesamtjahr 2010 rechnet der Schätzerkreis der Bundesregierung jedoch mit einer Unterdeckung der Kassenausgaben in Höhe von 3,1 Milliarden Euro.

Bei der Betrachtung der Finanzentwicklung im Jahresverlauf verwundert diese Schätzung allerdings, denn die Ausgaben in den Monaten Januar bis Juni sind zwar regelmäßig niedriger als im Durchschnitt des Gesamtjahres, die Zuweisungen aus dem Gesundheitsfonds werden aber in monatlich gleichen Teilbeträgen an die Krankenkassen ausgezahlt. Der Gesundheitsfonds zahlte für das erste Halbjahr 2010 Zuweisungen in Höhe von insgesamt rd. 85,16 Mrd. Euro an die Krankenkassen aus und somit 50\% des den Krankenkassen zugesicherten Jahresbedarfs. Die Einnahmen des Gesundheitsfonds aus Beiträgen und Bundeszuschüssen lagen bei 85,33 Mrd. Euro. In der zeitlichen Abgrenzung für das erste Halbjahr 2010 weist der Gesundheitsfonds deshalb einen Überschuss von 147 Mio. Euro aus. Die Leistungsausgaben der Krankenkassen sind im 1. Halbjahr 2010 um 4,2\% gestiegen. Der Schätzerkreis ist bei seiner letzten Jahresprognose von einem Anstieg von rd. 4,9\% ausgegangen. In den größeren Leistungsbereichen ist die Entwicklung der Ausgaben sehr unterschiedlich verlaufen: Der Zuwachs von 5,4\% bei den Ausgaben für ambulante ärztliche Behandlung nach einem Zuwachs von 7,4\% im gesamten Jahr 2009 zeigt, dass sich die Honorarsituation für die Ärzte auch in diesem Jahr verbessern wird.

\section{MMW- Kommentar}

Kritisch ist bei dem Ausgabenanstieg die Verteuerung bei den Ausgaben für die Krankenhausbehandlung von 4,2\% nach
6,6\% im Vorjahr zu sehen. Auch der Anstieg bei den Arzneimittelausgaben (ohne Impfkosten) lag bei $r d .4,8 \%$, womit sich der hohe Ausgabenanstieg der letzten Jahre nahezu unvermindert fortsetzt. Der Ausgabenzuwachs beim Krankengeld ist mit einem erneuten Plus von $10 \%$ nach den zweistelligen Zuwachsraten der Jahre 2008 und 2009 unverändert hoch. Hierzu tragen insbesondere eine zunehmende Zahl von Krankengeldberechtigten bei steigendem Renteneintrittsalter sowie eine starke Zunahme langwieriger psychischer Erkrankungen bei.

Skandalös sind die Zuwächse bei den Verwaltungskosten der Kassen. Die Nettoverwaltungskosten stiegen um $8,0 \%$. Fast hat es dabei den Anschein, dass sich die Kassen damit auf die gesetzliche Begrenzung der Verwaltungskosten der Kassen in den Jahren 2011 und 2012 auf das Niveau des Jahres 2010 vorbereitet haben. 